\title{
Fault Detection and Diagnosis System in Process industry
}

\section{Based on Big Data and WeChat}

\author{
Zengqiang Sun ${ }^{*}$ and Xinjian Lu \\ Nanjing ChemCyber Technology Company LTD, nanjing 210036, PR china
}

\begin{abstract}
The fault detection and diagnosis information in process industry can be received, anytime and anywhere, based on bigdata and WeChat with mobile phone, which got rid of constraints that can only check Distributed Control System (DCS) in the central control room or look over in office. Then, fault detection, diagnosis information sharing can be provided, and what's more, fault detection alarm range, code and inform time can be personalized. The pressure of managers who worked on process industry can be release with the mobile information system.
\end{abstract}

\section{Introduction}

The fault detection and diagnosis system of the process industry can be analyzed from diagnosis technology and communication technology. There are mechanism model, empirical model and large data model in diagnostic technology, in traditional model, which is based on the mechanism of the process and the artificial experience of the accident report [1]. But, people could acquire different experience from the same accident, because of the choice of observation parameter. In another side, the utilization of on-line mechanism model is too low. So, the large data model is applied more and more.

On communication technology, the detection and diagnosis information could be received only through DCS in the control room many years ago, the operator and administrator can't receive production and fault information promptly and automatically after leaving the control room. Then, the short message service (SMS) was used to notice fault information to administrator of the factory promptly and automatically with the SMS link to DCS, but the shortcomings are also obviously, such as the information is incomplete, usually need to make a telephone call for fault confirmation after receiving the information. With the development of mobile phone network, E-mail notification on phone was used for fault information communication also, but the continuity of the information can't meet need in production. In general, the above means of communication could not offer the fault detection and diagnosis information anytime and anywhere, so the safety pressure in process industry can't reduce effectively. But, the ease of use and continuous information of WeChat will offer a new way.

In this paper, a fault detection and diagnosis model based on big data will be describes and the communication means of the system will be show. The structure is organized as follows: Section 2 presents the bigdata model, and describes the display of system. Section 3 describes the precondition, technical route, Algorithm and case of system implementation. Finally, Section 4 concludes the paper and points out further work.

\section{The structure of system}

\subsection{The bigdata model of system}

In each target operation unit or device which selected for bigdata modeling, historical data that including normal production and fault status are scan at first, then, key performance indicators are select, and the model is established after principal component analysis (PCA) [2], squared prediction error (SPE) and $\mathrm{T}^{\wedge} 2$ detection [3-5]. So, the fault data in process can be pick out with the control line of parameter after the online data directly into the model, and diagnose information can be obtain according to different parameter's contribution on the control line indicators.

The fault data can be identified from process data at a certain time in accident analysis, but, from the point of large data statistical analysis, there are two kinds of identified methods in the process of fault occurrence. one is the deviation of the parameter itself, in other word, the parameters that trigger the fault will deviate from the stable data set when the fault occurs. The other is relevance of the different parameter, which will change from stable conditions to fault conditions states.

Big data modeling is a process to find the regularity implied in numerous data initiative, which is quantitative. 
And then the model will be confirmed by expert, which part is qualitative. As the artificial confirmation is based on big data, so the model is more accurate. On the other hand, the small data modeling is a process to find the regularity from human experience, which is qualitative. And then the model will be confirmed by small data case, which part is quantitative. Therefore, large data modeling can minimize the impact of human subjective factors, making the model more comprehensive, detailed and reasonable in fault detection and diagnosis.

\subsection{The display on Wechat of system}

The fault detection and diagnosis information pushed by the system can meet the needs of the user anytime and anywhere, the content is more complete and continuous with the trend graph of the production data when the fault occurs.

The system will not meet the requirements of safe production if only one person to view the fault alarm information, the safety accident caused from someone negligence is not uncommon. But, WeChat will share the fault alarm information to different levels of management personnel. On the one hand, fault knowledge base or parameter model can be established early. On the other hand, fault Information can be diagnosed after expert consultation, and the system will update the diagnostic information to the knowledge base and parameter model, so the model can be improved continuously.

The manager who works on different positions pay different attention for different fault information, therefore, everyone should have the authority to adjust whether they are concerned about the fault information and the scope of the fault alarm. Such as the fault have more impact of your work should be adjust to a narrow alarm range, so that appropriate measures can be ready for the fault. In another way, the authority to adjust system personalized which keep operators and managers have a good rest.

As above characteristics, the comparison between SMS and WeChat communication system as follows:

Table 1. Comparison between SMS and Wechat

\begin{tabular}{|c|c|c|}
\hline timeliness & $\begin{array}{c}\text { Immediate } \\
\text { arrival }\end{array}$ & $\begin{array}{c}\text { Immediate } \\
\text { arrival }\end{array}$ \\
\hline Graphic display & No & Yes \\
\hline Historical inquiry & No & Yes \\
\hline Correlation analysis & No & Yes \\
\hline personalization & Few & More \\
\hline User number limit & Yes & No \\
\hline
\end{tabular}

\section{The implementation of system}

\subsection{Precondition}

The fault detection and diagnosis system based on large data and WeChat relies on real-time data at first, so that DCS, real-time database (RTD) or other control system are required. Then, real-time data should also be allowed to transport with Internet transmission or intranet transmission. Of course, the transmission process is security by the certificate and encrypted channel. In comparison, the cost, storage and computing of Internet transmission is lower and convenient.

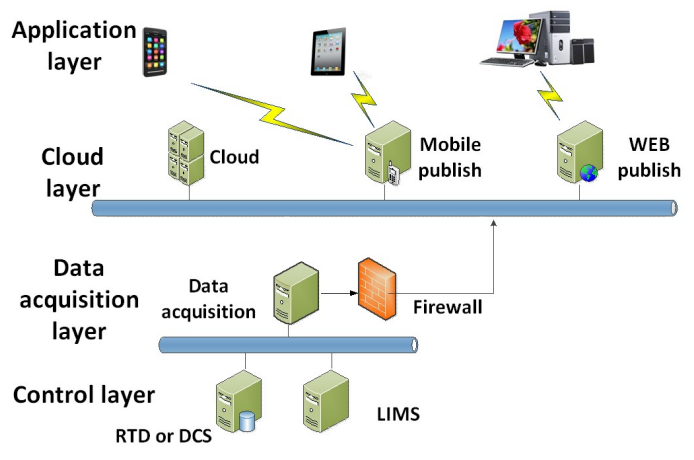

Fig. 1. Network topology diagram.

\subsection{Technical route}

Data acquisition safely is the first step for system implementation, so the acquisition device should be Connect to DCS or real-time database in a secure manner, such as adding a firewall or gatekeeper to ensure that the data does not leak or prevent the DCS from reversing control. In addition, the acquisition device could not affect the DCS or other information systems connected to the real-time database.

Data pretreatment can be used for increasing the safety in data transmission to the cloud servers, for example, data and corresponding physical explanation can be separate in transmission by pretreatment, so someone who gets the data through informal channels can do nothing without physical explanation. In another case, the data was deal by addition or multiplication before transmission to the cloud servers for safety, and would be restoration for modeling.

Real-time data could be stored to the cloud servers, which reduce the physical risk and cost of enterprises to buy physical storage servers. Then, the managers have no need to worry about power outages, host computer server crash and data disaster, because a professional team of cloud server suppliers to maintain. The last, realtime data of every enterprise in the cloud servers are stored separately, other enterprises have no right to access.

Model calculation, especially for high frequency calculation is a key step for real-time detection, the system achieves that through distributed and parallel cloud computing, in which computing tasks are distributed across resource pool of cloud servers that 
constitute from a lot of computers, rather than local computers or remote servers. So, computational power, storage space, and information services of various application functions can to obtain. Moreover, the available resources are infinite expansion and can be readily available at any time.

In addition, the storage and calculation of data must follow a clear classification and unified data structure. So that users can easily obtain the required data. These classification and structure are also base for fault detection and diagnosis system.

The user can receive fault detection and diagnosis messages with pay close attention and register to system on WeChat, after the calculation result in cloud servers is published to the WeChat servers.

The roadmap of data flow as follows:

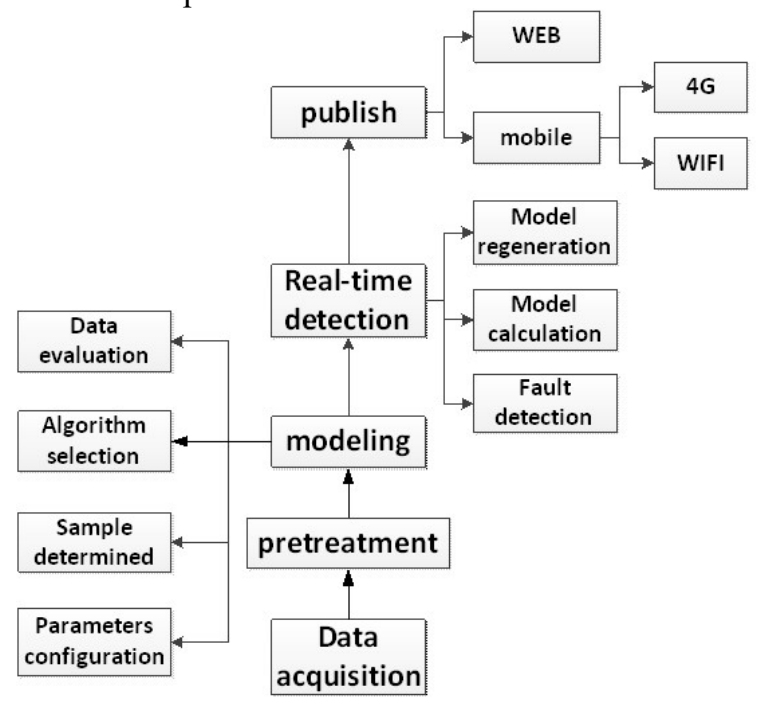

Fig. 2. Roadmap of data flow.

\subsection{Algorithm}

The basic algorithm used in the system, including principal component analysis and other statistics classical method, in addition, SPE and $\mathrm{T}^{\wedge} 2$ detection which used for fault detection and diagnosis. In this paper, these algorithms and some data pretreatment are combined to enhance the practicality of the results.

Steps are as follows:

(1) Collecting data from the real-time database to form a sample set for modeling;

(2) Extracting the key performance parameters which according to the physical model to form a new sample set for one specific operating unit;

(3) Normalizing the key performance parameters of the new sample set;

(4) Executing PCA of the normalized set of samples;

(5) Modeling with the lower limit or control line of SPE and $\mathrm{T}^{\wedge} 2$ when the confidence is $95 \%$. The fault was identified when the value of real-time data about parameters are significantly higher than the two lower limits at the same time, such as 3 times of standard deviation of lower limit according to score matrix obtained by PCA.
(6) Comparing the contribution of different parameters to $\mathrm{SPE}$ and $\mathrm{T}^{\wedge} 2$, then the parameter which caused a malfunction could be diagnosis.

\subsection{Case}

Figure 3 happened at 10:43 on March 30, 2015, the DCS showed the flow value of non-purified wind which into the device of one enterprise in the normal range, but the instantaneous deviation was abnormal, as the point of blue data trend line breakthrough the red control line in figure 3. The operator didn't take anything action on DCS, but, the detection information from our system was sent to the technical staff, and the technical staff entered site for inspection, then, found a hidden danger. The parameter returned to normal after the hidden danger was elimination. If not, there would be a great impact on pipe network system, even device shutdown.

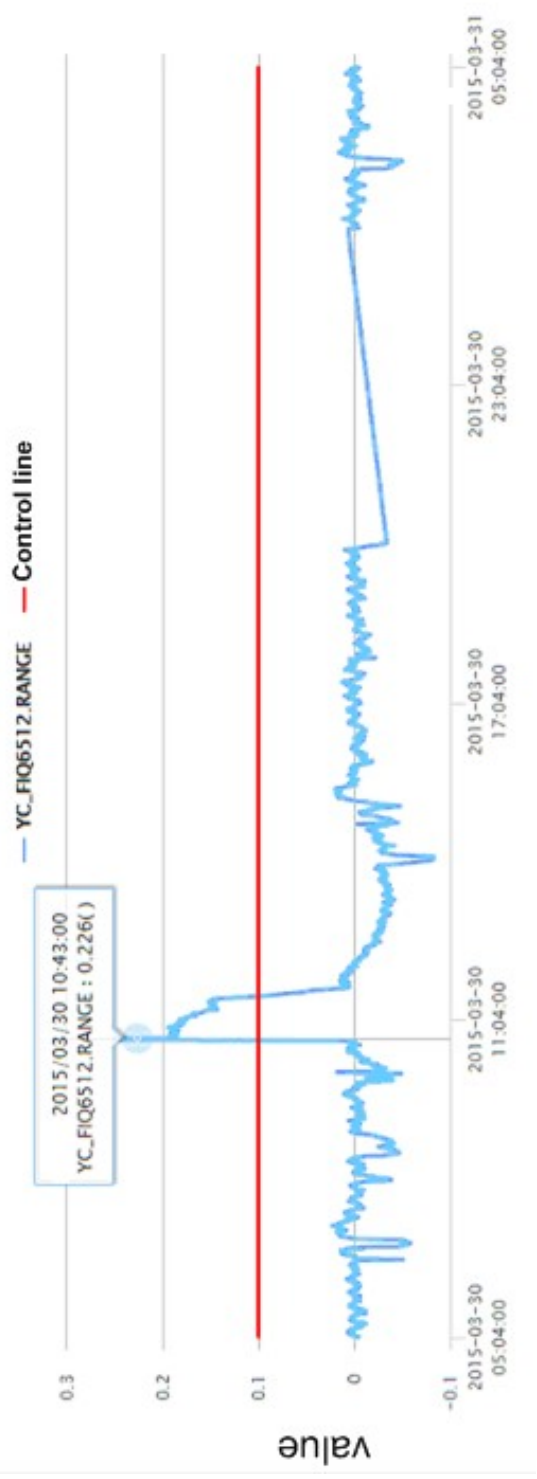

Fig. 3. Case 1 of data system

Figure 4 happened at $12: 10$ on January 12,2015 , the DCS showed a rapid decline of the external heaters material level, and then quickly restore the phenomenon, which didn't trigger alarm on DCS, so the operator 
didn't take more care of that. But our system diagnosed there would be a fault of external heaters leak according to the comprehensive detection of relevant parameters, as the external heaters comprehensive detection parameters breakthrough the normal value obviously, although external heaters material level parameters was normal. Then sent the fault message to the technical staff, and the technical staff entered site for inspection of tube bundle in junction box of import and export on external heating device, then, found the tube $2 \#$ had leakage. The device returned to be normal after the tube $2 \#$ had be resection.

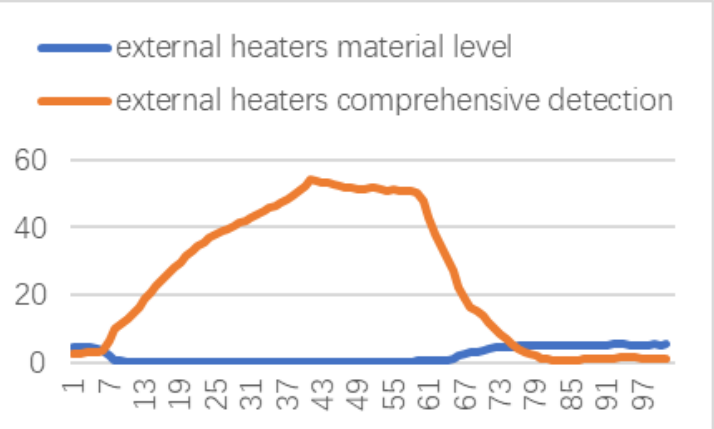

Fig. 4. Case 2 of data system

There would be a difficult for operator or technical staff to judge the fault manually, because of the not obvious of DCS data abnormal and then quickly restored. If not promptly dealt with, which could cause the regenerator pressure too high, affected the normal circulation of the catalyst, even lead to smoke machine vibration increasing if seriously, caused the smoke machine damage, and even caused the reaction feed was cut off, finally to the device emergency shutdown, which need 6 hours to recover and avoid the loss of 2.39 million yuan.

\section{Conclusions}

This paper introduces the fault detection and diagnosis system based on large data and WeChat developed by Nanjing ChemCyber Technology Co., Ltd., including technology route and algorithm realization, and provides the realization case. The system reduces the safety pressure of manager in process industry, so that safety management more convenient. And the continuous optimization of the device can be implementation with fault diagnosis information, so that the process industry can enjoy the changes and benefits by mobile information system.

\section{References}

1. L. Zhou, Z.H. Song, B.P Hou, Z.X. Fei. CIESC Journal, 68, (2017), 1109-1115.

2. M. Han, Z.K. Zhang. CIESC Journal, 66, (2015), 2139-2149,

3. K.X. Peng, L. Ma, K. Zhang, ACTA AUTOMATICA SINICA, 43, (2017), 349-365

4. B. Song, Y.X. Ma, Y.F. Fang, H.B Shi. CIESC Journal, 65, (2014), 620-627
5. A Carolos, M.A. Francisco, Q.C. Eduardo, G.M. Emilio, Journal of Loss Prevention in the process industries, 26, (2013), 660-665 